\title{
COMPULSÃO ALIMENTAR EM PACIENTES NO PRÉ-OPERATÓRIO DE CIRURGIA BARIÁTRICA
}

\section{Binge-Eating in Pre-Operative Patients after Bariatric Surgery}

\author{
Magda Rosa Ramos Quadros ${ }^{1}$ \\ Giovanna Teresa Bruscato ${ }^{2}$ \\ Alcides José Branco Filho ${ }^{3}$
}

\section{Resumo}

Com o objetivo de verificar a prevalência de compulsão alimentar em obesos mórbidos durante o período pré-operatório de cirurgia bariátrica, aplicou-se o questionário de Escala de Compulsão Alimentar Periódica, em 30 pacientes atendidos no Ambulatório de Cirurgia Bariátrica da Santa Casa de Misericórdia da PUCPR, no período de abril e maio de 2005. Do total de pacientes, 26 eram do sexo feminino e 4 do sexo masculino. Verificou-se que $53,2 \%$ dos entrevistados apresentaram compulsão alimentar moderada ou grave, sendo que $25 \%$ dos pacientes com obesidade mórbida apresentaram compulsão alimentar grave. O IMC médio dos pacientes foi de $51,26 \mathrm{~kg} / \mathrm{m}_{-}$, sendo de 38,4 e de $50,44 \mathrm{~kg} / \mathrm{m}_{-}$, para pacientes com obesidade moderada e grave, respectivamente. Quando aplicado o teste Qui-quadrado, não foi encontrada correlação significativa entre IMC e grau de compulsão alimentar $(\mathrm{p}>0,05)$. Com este estudo, conclui-se que os pacientes obesos mórbidos apresentam maiores índices de compulsão alimentar do que pacientes obesos, mas nada teve a ver o grau de compulsão com o IMC. O acompanhamento psicológico é de suma importância para a boa evolução do paciente, bem como o acompanhamento nutricional. Estudos futuros serão necessários para melhor compreensão do quadro do paciente, sendo objetivo, para um próximo trabalho, a re-avaliação deles quando estes estiverem com 6 meses ou mais de pós-operatório para verificar a evolução do quadro.

Palavras-chave: Compulsão alimentar; Obesidade mórbida; Cirurgia bariátrica.

\section{Abstract}

Verifying the prevalence of binge eating in morbid obeses during the bariatric surgery pre-operative period, a questionnaire was applied in the Periodic Binge Eating Scale, in thirty patients in the bariatric surgery ward atSanta Casa de Misericóndia/PUCPR, from April to May, 2005. From all the patients, 26 was female and 4 male. It was verified that $53,2 \%$ of the patients presented binge eating moderate or severe. The average BMI of the patients was $51,26 \mathrm{~kg} / \mathrm{m}_{-}$, being 38,4 and $50,44 \mathrm{~kg} / \mathrm{m}_{-}$, for patients with moderate and severe obesity, respectively. When the Qui-quadrado Test was applied, a meaningful correlation was not found between the BMI and level of binge eating $(p>0,05)$. Using this work It was concluded that the morbid obese patients presented higherindexes of binge eating than the obese patients. Nothing else is related to the binge eating level with the BMI. The psychological assistance is fundamental to follow better patient's behavior, as well as, the nutritional assistance. Further researches will be necessary for a better understanding about the patient's situation, aiming for the next work, a re-evaluation of the patients in post-operative sixth months or more, to verify its evolution.

Keywords: Binge eating; Morbid obese; Bariatric surgery.

\footnotetext{
1 Professora do Curso de Nutrição, CCBS, PUCPR; especialista em Nutrição Clínica pela UFPR; mestranda em Tecnologia em Saúde na PUCPR; responsável pelo serviço de nutrição do CEVIP (Centro de Videolaparoscopia do Paraná). End. Rua Alberto Stenzowski, 110, apto. 144, Novo Mundo, Curitiba-PR, CEP: 81050-020 - magda.quadros@pucpr.br

2 Aluno do Curso de Nutrição, CCBS, PUCPR.

3 Cirurgião responsável pelo ambulatório de nutrição da Santa Casa/PUCPR e pelo CEVIP.
} 


\section{Introdução}

Cirurgia Bariátrica é o nome dado às intervenções realizadas no aparelho digestivo para tratamento da obesidade e tem como objetivo promover a redução do peso. Candidatos a essa cirurgia são pacientes que apresentam obesidade mórbida, ou seja, índice de massa corporal (IMC) acima de 40, ou pacientes com IMC entre 35 e 40, que manifestam alguma patologia associada capaz de melhorar com a redução de peso, como: hipertensão arterial, diabetes, apnéia do sono, problemas ortopédicos, entre outras (Cowan Jr, Hiler, \& Buffington, 2000; Mahan \& Escott-Stump, 2003).

A obesidade é um excesso de gordura corporal que freqüentemente causa danos à saúde. Ela é tratada como doença, pois diminui a qualidade e a expectativa de vida. Algumas causas da obesidade são: fatores genéticos, problemas psicológicos, transtornos alimentares, distúrbios endócrinos e metabólicos, má alimentação e sedentarismo. Dentre os transtornos alimentares que levam à obesidade, destaca-se a compulsão alimen$\operatorname{tar}$ (Segal \& Fandiño, 2002).

Os transtornos alimentares são doenças psiquiátricas caracterizadas por alterações dramáticas do comportamento alimentar. São caracterizados pela prática de dietas restritivas e aleatórias, uso de produtos dietéticos sem recomendação e uso de métodos inadequados para perda e manutenção de peso (Borges, 2001).

O Transtorno da Compulsão Alimentar Periódica (TCAP) é uma nova categoria diagnóstica de transtorno alimentar, descrita no Apêndice B do DSM IV (Manual de Diagnóstico e Estatística das Perturbações Mentais) entre as categorias em fase de pesquisa. Este transtorno define-se basicamente pela compulsão alimentar, ou seja, por ingestão de grandes quantidades de comida associada à perda de controle, na ausência de métodos apropriados para controle de peso.

Vários estudos demonstram que o TCAP é freqüente entre obesos e dificulta o tratamento para a perda de peso. Indivíduos obesos com este diagnóstico diferenciam-se de obesos sem transtorno alimentar por apresentarem obesidade mais precoce, freqüentes oscilações de peso, índices mais elevados de depressão e ansiedade (Borges, 2001).

O TCAP encontra-se, até o presente momento, entre os "transtornos alimentares sem ou- tra especificação" e sua caracterização baseia-se na presença de compulsão alimentar, com subseqüente angústia por tal comportamento (Borges \& Jorge, 2000).

Devlin, Goldfein, e Dobrow (2003) abordaram o estado atual da nosologia do TCAP resumindo exaustivamente as evidências que pudessem apoiar ou refutar quatro formas de conceituálo. No primeiro modelo conceitual, o TCAP é considerado um subtipo distinto de transtorno alimentar; no segundo, um subtipo de Bulimia Nervosa; no terceiro, é visto como um subtipo comportamental de obesidade; e no último modelo hipotético, o TCAP é considerado uma condição clínica que ocorre quando dois transtornos primários coexistem (a obesidade e a depressão, ou a obesidade e a impulsividade). No final do artigo, os autores concluem que, baseando-se nas evidências atuais, nenhum destes modelos poderia ser excluído completamente.

A compulsão alimentar enquanto comportamento observado entre obesos foi descrita pela primeira vez por Stunkard (1959), no sentido de uma ingestão de enormes quantidades de comida em um curto espaço de tempo, seguida por sentimentos de desconforto físico e de autocondenação.

O comportamento de compulsão alimentar, ou em inglês "binge eating", afeta geralmente, pessoas obesas e que apresentam uma história de variação de peso, pois a comida é usada para lidar com problemas psicológicos (Appolinário, 2004).

Segundo o DSM IV (1994), um episódio de compulsão periódica é caracterizado por ambos os critérios:

1- ingestão, em um período limitado de tempo (dentro de 2 horas, por exemplo), de uma quantidade de alimento definitivamente maior do que a maioria das pessoas consumiria em um período similar, sob circunstâncias similares;

2- um sentimento de falta de controle sobre o consumo alimentar durante o episódio, ou seja, sentimento de não conseguir parar ou controlar o que ou quanto se está comendo naquele momento.

Para caracterizar o TCAP, deve-se associar três ou mais dos seguintes critérios:

1- comer muito mais rapidamente do que o normal;

2- comer até sentir-se incomodamente repleto; 
3- comer grandes quantidades de alimentos, quando não fisicamente faminto;

4- comer sozinho, em razão do embaraço pela quantidade de alimentos que consome e

5- sentir repulsa por si mesmo, depressão ou demasiada culpa após comer excessivamente.

A compulsão periódica ocorre, em média, 2 vezes por semana, por 6 meses, para que se possa caracterizá-la e não está associada com 0 uso regular de comportamentos compensatórios inadequados.

A compulsão alimentar vem sendo relacionada à baixa produção de serotonina (uma substância química encontrada no cérebro, que causa sensação de bem-estar). Medicamentos que aumentam os níveis de serotonina e prática de atividade física reduzem a ocorrência de episódios compulsivos. Já situações de ansiedade, depressão ou dietas muito rígidas com poucos carboidratos tendem a aumentar a compulsão (Fandiño, Benchimol, Couttinho, \& Appolinário, 2004).

A obesidade severa ou mórbida é uma doença grave, o seu impacto na sociedade, as repercussões na qualidade e a diminuição no tempo de vida dessas pessoas são razões mais do que suficientes para justificar os atuais critérios de intervenção para amenizar o problema (Porto, Brito, \& Calfa, 2002).

Sabe-se que esta patologia favorece 0 aparecimento de outras doenças associadas, tais como hipertensão arterial sistêmica, diabetes mellitus do tipo 2, dislipidemia, angina, infarto do miocárdio, doença articular, apnéia do sono, câncer, problemas ortopédicos, doenças cardiovasculares, morte súbita e outras comorbidades (Csendes et al., 1999).

Para os casos mais graves de obesidade mórbida asso ciada a um quadro de compulsão alimentar incontrolável, que não melhora com uso de medicamentos, existe o recurso da cirurgia bariátrica. Uma pesquisa realizada na Itália demonstrou uma nítida melhora da compulsão alimentar em pacientes no pós-operatório (Oliveira, Linardi, \& Azevedo, 2004), o que pode ser considerado mais um benefício para o paciente; porém um acompanhamento psicológico rigoroso faz-se necessário para que o paciente possa apresentar boa evolução.

Para que este possa se submeter à cirurgia bariátrica, é necessária uma avaliação por uma equipe multidisciplinar composta por: cirurgião, cardiologista, pneumologista, nutricionista, psicólogo e outros profissionais, se houver necessidade (Cruz \& Morimoto, 2004).

O nutricionista, por meio de uma anamnese nutricional detalhada, verifica os hábitos alimentares do paciente prescrevendo-lhe uma dieta hipocalórica, visando uma perda de peso, na fase pré-cirúrgica, entre 5 a $10 \%$, com o objetivo de melhorar a sua capacidade respiratória no pósoperatório imediato (Repetto, Mottin, Casagrande, $\&$ Marchese, 2001).

O papel do nutricionista nesse tipo de cirurgia é de suma importância, pois facilita a aquisição de novos hábitos alimentares, mas para isso se faz necessária a adesão do paciente ao processo de reeducação alimentar.

Para diagnóstico do TCAP e classificação do grau apresentado pelo paciente, é utilizada a Escala de Compulsão Alimentar Periódica, do inglês Binge Eating Scale, a qual é considerada para uso clínico (Freitas, Lopes, \& Couttinho, 2001).

Esta será utilizada com o intuito de verificar a prevalência de compulsão alimentar em pacientes obesos mórbidos, durante o período préoperatório de cirurgia bariátrica.

Dentre os objetivos deste estudo estão: definir o percentual de pacientes com transtorno da compulsão alimentar no pré-operatório e verificar o grau de compulsão apresentado; verificar o IMC médio; correlacionar o IMC com o grau de compulsão alimentar; verificar o número de tratamentos feitos para perda de peso e o número de pacientes em acompanhamento com a psicologia e/ou psiquiatria neste período.

\section{Métodos}

O estudo foi de caráter quantitativo onde se aplicou o questionário de Escala de Compulsão Alimentar Periódica, com a finalidade de identificar se os pacientes em preparo pré-cirúrgico de cirurgia bariátrica apresentam quadro de compulsão alimentar e a gravidade desta.

Foram incluídos no estudo todos os pacientes atendidos pelo serviço de nutrição do Ambulatório de Cirurgia Bariátrica da Santa Casa/PUCPR, durante o período de abril e maio de 2005, em acompanhamento nutricional pré-operatório de cirurgia bariátrica. 
A técnica cirúngica realizada neste ambulatório é a de Fobi-Capella (técnica mista), que se caracteriza por restrição do volume alimentar pela redução do estômago e redução da absorção de nutrientes pelodesvio intestinal.

Os pacientes foram informados quanto aos objetivos da pesquisa e assinaram o termo de consentimento livre e esclarecido quando de acordo, podendo parar a pesquisa a qualquer momento, sem prejuízo no tratamento.

Não foram incluídos no trabalho os pacientes que optaram por não participar da pesquisa e todos que já se submeteram à cirurgia.

Foram coletados dados referentes ao número de tratamentos para perda de peso, acompanhamento psicológico e/ou psiquiátrico e dados antropométricos.

Quanto aos dados antropométricos foram coletados: peso, medido na balança digital da marca Filizola, a qual comporta até $250 \mathrm{~kg}$, onde os pacientes foram pesados sem calçado e vestindo roupas leves; altura, medida no estadiômetro da marca Seca, onde os pacientes foram medidos descalços, encostados na parede; e cálculo do IMC pela fórmula: $\mathrm{IMC}=\mathrm{Peso} /$ altura $^{2}$.

Dados quanto ao acompanhamento psicológico e/ou psiquiátrico e o número de trata- mentos realizados para perda de peso foram coletados por entrevista individual, realizada pela estagiária do serviço de nutrição.

Para verificar a correlação entre o IMC e o grau de compulsão, foi utilizado o teste Quiquadrado $(\mathrm{p}<0,05)$.

Os termos de consentimento livre e esclarecido, bem como os questionários aplicados, estão arquivados no Ambulatório de Cirurgia Bariátrica da Santa Casa/ PUCPR.

Este estudo foi submetido à avaliação do comitê de ética em pesquisa da PUCPR e aprovado pelo Of. 131/05/CEP-PUCPR, no dia 05/05/05.

\section{Resultados e Discussão}

Foram entrevistados 30 pacientes, 26 do sexo feminino e 4 do sexo masculino.

Dos pacientes analisados neste estudo, 8 apresentaram compulsão alimentar grave, 8 apresentaram moderada e 13 apresentaram compulsão leve ou não apresentaram, segundo a classificação da escala de compulsão alimentar periódica.

O gráfico I mostra os níveis de compulsão apresentados por pacientes obesos mórbidos no pré-operatório de cirurgia bariátrica.

\section{Gráfico I. Nível de Compulsão Alimentar segundo a Escala de Compulsão Alimentar Periódica.}

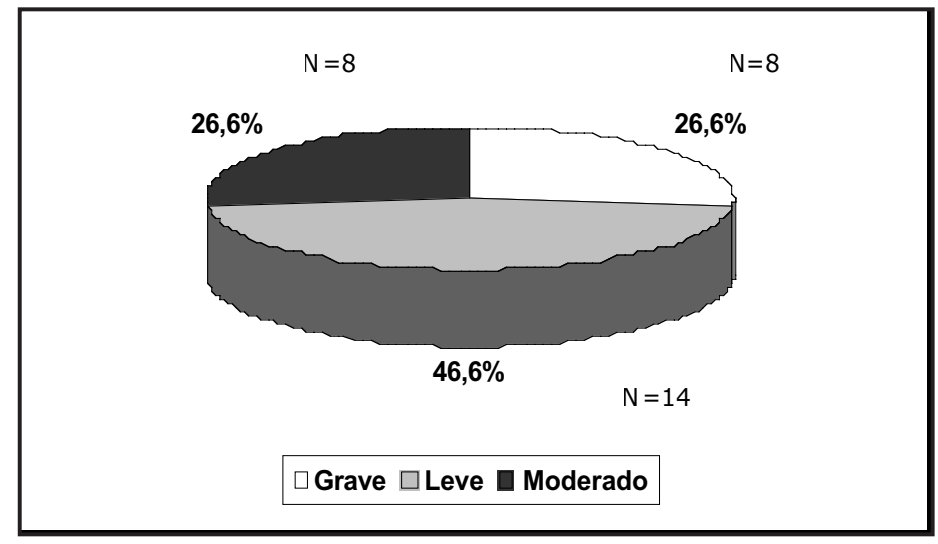


Segundo Appolinário (2004), enquanto a prevalência estimada de TCAP na população geral pode variar de 1,5 a $5 \%$, em amostras clínicas de pacientes obesos encontram-se em torno de 7,5 a $30 \%$.

De acordo com Oliveira et al. (2004), a prevalência de compulsão alimentar na população obesa mórbida é de $1 / 3$ à metade.

Pode-se observar que, neste estudo, 53,2\% dos pacientes apresentaram diagnóstico de TCAP moderada ou grave, ou seja, quando analisada a população de obesos mórbidos, o número de pacientes com o transtorno é realmente superior ao encontrado em obesos, confirmando a afirmação dos autores acima.

Dos pacientes analisados, 3 (10\%) pacientes foram classificados com quadro de obesidade moderada, isto é, IMC entre 35 e 40, e 27 (90\%) foram classificados como obesidade grave, IMC acima de 40. Na tabela I, estão os dados referentes aos IMC encontrado.

\section{Tabela I - IMC médio}

\begin{tabular}{|l|l|l|l|}
\hline & Número de Pacientes & IMC Médio & Desvio Padrão \\
\hline Obesidade Moderada & $\mathrm{n}=3$ & 38,40 & $\pm 5,81$ \\
Obesidade Grave & $\mathrm{n}=27$ & 51,26 & $\pm 8,12$ \\
\hline Total & $\mathbf{n}=\mathbf{3 0}$ & $\mathbf{5 0 , 4 4}$ & $\pm \mathbf{6 , 9 6}$ \\
\hline
\end{tabular}

O IMC médio dos pacientes que participaram do estudo foi de $50,44 \mathrm{~kg} / \mathrm{m}_{-}$, confirmando a indicação cirúrgica para tratamento da obesidade.

Em pesquisa brasileira, coordenada por Borges (2001), realizada com obesos que participavam do Programa Vigilantes do Peso, notou-se que dos distúrbios alimentares mais comuns, o que apresentou maior incidência foi a compulsão alimentar (43\%).

Na tabela II estão os dados referentes ao grau de compulsão alimentar relacionado com 0 IMC apresentado por ele.

\section{Tabela II - Grau de compulsão a limentar e IMC do paciente.}

\begin{tabular}{|l|l|l|l|l|}
\hline \multirow{2}{*}{ IMC } & \multicolumn{3}{|c|}{ Grau de compulsão alimentar } & \\
\hline & Grave & Moderado & Leve ou ausente & Total \\
\hline Ob. Grave & 7 & 8 & 12 & 27 \\
Ob. Moderada & 1 & 0 & 2 & 3 \\
\hline Total & $\mathbf{8}$ & $\mathbf{8}$ & $\mathbf{1 4}$ & $\mathbf{3 0}$ \\
\hline
\end{tabular}

De acordo com Fandiño et al. (2004), a compulsão alimentar periódica é uma síndrome psiquiátrica freqüentemente encontrada na população pré-operatória de cirurgia bariátrica, devido a sua associação com a obesidade.

Adami et al. (1995) demonstraram, por meio de uma avaliação transversal no período préoperatório, que $63 \%$ dos pacientes com obesidade mórbida apresentavam compulsão alimentar periódica grave. Estas taxas foram muito maiores do que aquelas observadas em pacientes sem obesidade mórbida.

Analisando os resultados obtidos pelo questionário aplicado, observou-se que 25\% dos pacientes com obesidade mórbida apresentaram compulsão alimentar grave, o que não está de acordo com os estudos realizados sobre o assunto.

Quando aplicado o Teste Qui-quadrado, não foi encontrada correlação significativa entre 0 IMC e o grau de compulsão alimentar apresentado 
pelo paciente $(p>0,05)$, não sendo possível qualquer associação entre o excesso de peso e o grau de compulsão alimentar.

Este fato pode indicar uma dificuldade dos pacientes que apresentam IMC mais altos em aceitar a doença (TCAP) e seus sintomas, bem como, de expor ao profissional a sua relação com os alimentos devido ao caráter compulsivo que esta apresenta e a sua consciência de que este comportamento não é adequado.
A média de números de tratamentos realizados para emagrecer sem sucesso por paciente foi de 12,07, com Desvio Padrão de $\pm 8,14$. De acordo com o NIH (2000), estima-se que $80 \%$ das pessoas que perdem peso o recuperam, e entre $1 /$ 3 a 2/3 desta recuperação ocorre logo no primeiro ano após a perda de peso. Por esse motivo, observa-se 0 alto índice de tentativas frustradas para perda de peso nesses pacientes.

Na tabela III estão os dados referentes ao acompanhamento psicológico e psiquiátrico.

\section{Tabela III - Número de pacientes em tratamento psicológico e/ou psiquiátrico}

\begin{tabular}{|l|l|l|}
\hline & $\mathbf{N}$ & $\mathbf{\%}$ \\
\hline Acompanhamento psicológico & 22 & 73 \\
Acompanhamento psiquiátrico & 0 & 0 \\
Acompanhamento psicológico e psiquiátrico & 8 & 27 \\
Pacientes sem tratamento & 8 & 27 \\
\hline
\end{tabular}

Dentre os entrevistados, $73 \%$ fazem apenas acompanhamento psicológico; $27 \%$ fazem acompanhamento psicológico e psiquiátrico, não havendo pacientes apenas em acompanhamento psiquiátrico; e 27\% dos pacientes não fazem tratamento.

Esse tipo de acompanhamento é de suma importância, pois conforme cita Hsu, Betancourt, \& Sullivan (1996), há um considerável número de literatura indicando que comedores compulsivos são um subgrupo distinto da população obesa que apresentam mais sintomas psiquiátricos comparado com pessoas com sobrepeso ou obesidade que não apresentam compulsão alimentar, e que esses apresentam também mais distúrbios psicológicos, incluindo depressão e ansiedade, desistindo do tratamento para perda de peso mais facilmente e assim recuperando mais rapidamente o peso perdido.

\section{Conclusão}

Com este estudo, conclui-se que os pacientes obesos mórbidos apresentam maiores índi- ces de compulsão alimentar do que pacientes obesos, porém, não é possível afirmar que o grau de compulsão está relacionado com o IMC.

Considerando o número de tentativas frustradas para emagrecer, o IMC apresentado por esta população e 0 alto índice de pacientes com TCAP, pode-se afirmar que a cirurgia da obesidade é para estes pacientes a última alternativa de tratamento para redução de massa corporal.

Fica claro também que a presença de distúrbios psicológicos diminuem a adesão destes pacientes ao tratamento para perda de peso a longo prazo, seja por omissão do que realmente sentem, vergonha, desprezo ou baixa autoestima, o que reforça a importância do processo de reeducação alimentar e acompanhamento psicológico no pré e principalmente no pós-operatório de cirurgia bariátrica.

Um próximo estudo será a continuidade deste trabalho utilizando-se a mesma amostra de pacientes, já no período pós-operatório, para detectar se a compulsão alimentar continua, ou será amenizada nos mesmos pacientes. 


\section{Referências}

Adami, G. F., Gandolfo, P., Bauer, B., \& Scopinaro, N. (1995). Binge Eating im Massively Obese Patients Undergoingo Bariatric Surgery. Int. J. Eat. Disords, 17, 45 - 50.

Appolinário, J. C. (2004). Transtorno da Compulsão Alimentar Periódica: Uma Entidade Clínica Emergente que Responde ao Tratamento Farmacológico. Revista Brasileira de Psiquiatria, 26(2).

Borges, M. B. F. (2001). Estudo do transtorno da Compulsão Alimentar Periódica em População de Obesos e sua Associação com Depressão e Alexitimia. Psiquiatria na Prática Médica, 34.

Borges, M. B. F., \& Jorge, M. R. (2000). Evolução Histórica do Conceito de Compulsão Alimentar. Psiquiatria na Prática Médica, 33.

Cowan Jr, G., Hiler, L M., \& Buffington, C. K. (2000). Criteria for Selection of Patients for Bariatric Surgery. In Update: Surgery for the Morbidly Obese Patient (pp. 73-84). Toronto Canadá.

Cruz, M. R. R, \& Morimoto, I. M. I. (2004). Intervenção Nutricional no Tratamento Cirúrgico da Obesidade Mórbida: Resultados de um Protocolo Diferenciado. Revista de Nutrição - Brazilian Journal of Nutrition, 17, 263-272.

Csendes, A., \& al, e. (1999). Resultados Preliminares de la Gastroplastia Horizontal con Anastomosis em Y de Roux como Cirurgia Bariatrica en Pacientes con Obsidad Severa y Morbida. Revista Médica Chile, 127, 953-960.

Devlin, M. J., Goldfein, J. A., \& Dobrow, I. (2003). What is this Thing Called BED? Current Status of Binge Disorder Nosology. International Journal Disorders, 34.

Fandiño, J., Benchimol, A. K., Couttinho, W. F., \& Appolinário, J. C. (2004, janeiro abril). Cirurgia Bariátrica: Aspectos Clínico-Cinúngicos e Pciquiátricos. Revista de Psiquiatria, 26, 47-51.
Freitas, S., Lopes, C. S., \& Couttinho, W. F. (2001, dezembro). Tradução e Adaptação para o Português da Escala de Compulsão Alimentar Periódica. Revista Brasileira de Psiquiatria, 23, $215-220$.

Hsu, L. K., Betancourt, S., \& Sullivan, S. P. (1996). Eating Disturbances Before and After Vertical Banded Gastroplasty: A Pilot Study. Int. J. Eat. Disords, 19, 23 - 24.

Mahan, K. L, \& Escott-Stump, S. (2003). Alimentos, Nutrição e Dietoterapia (10 ed.). São Paulo: Editora Roca.

NIH, N. I. o. H., Institute, N. H. L. a. B., \& Obesity, N. A. A. f. t. S. o. (2000, outubro). The Pratical Guide: Identification, Evaluation, and Treatment of Overweight and Obesity in Adults. NIH Publication Number 00-4084.

Oliveira, M. V., Linardi, R. C., \& Azevedo, A. P. (2004). Cirurgia Bariátrica: Aspectos Psicológicos e Psiquiátricos. Revista de Psiquiatria Clínica, 31, 199 - 201.

Porto, M. C. V., Brito, I. C., \& Calfa, A. D. F. (2002). Perfil do Obeso Classe III do Ambulatório de Obesidade de um Hospital Universitário de Salvador, Bahia. Arquivo Brasileiro de Endocrinologia e Metabologia, 46(6), 668 - 673.

Repetto, G., Mottin, C. C., Casagrande, D., \& Marchese, C. (2001, maio/junho). Tratamento Clínico e Cirúrgico da Obesidade Mórbida. Revista Nutrição em Pauta.

Segal, A., \& Fandiño, J. (2002). Indicações e Contra-Indicações para Realização das Operações Bariátrica. Revista Brasileira de Psiquiatria, 24(3), 68 - 77.

Stunkard, A. J. (1959). Eating Patterns and Obesity. Psyquiatric Q, 33, 284 - 292.

Recebido em/received in: 14/03/2006 Aprovado em/approved in: 17/04/06 